\title{
Gray-Molasses Optical-Tweezer Loading: Controlling Collisions for Scaling Atom-Array Assembly
}

\author{
M. O. Brown, ${ }^{*}$ T. Thiele, ${ }^{*}$ C. Kiehl, T.-W. Hsu, and C. A. Regal ${ }^{\dagger}$ \\ JILA, National Institute of Standards and Technology and University of Colorado, \\ and Department of Physics, University of Colorado, Boulder, Colorado 80309, USA
}

(Received 21 November 2018; revised manuscript received 5 January 2019; published 29 March 2019)

\begin{abstract}
To isolate individual neutral atoms in microtraps, experimenters have long harnessed molecular photoassociation to make atom distributions sub-Poissonian. While a variety of approaches have used a combination of attractive (red-detuned) and repulsive (blue-detuned) molecular states, to date all experiments have been predicated on red-detuned cooling. In our work, we present a shifted perspective-namely, the efficient way to capture single atoms is to eliminate red-detuned light in the loading stage and use bluedetuned light that both cools the atoms and precisely controls trap loss through the amount of energy released during atom-atom collisions in the photoassociation process. Subsequent application of reddetuned light then assures the preparation of maximally one atom in the trap. Using $\Lambda$-enhanced graymolasses for loading, we study and model the molecular processes and find we can trap single atoms with $90 \%$ probability even in a very shallow optical tweezer. Using 100 traps loaded with $80 \%$ probability, we demonstrate one example of the power of enhanced loading by assembling a grid of 36 atoms using only a single move of rows and columns in 2D. Our insight is key in scaling the number of particles in a bottom-up quantum simulation and computation with atoms, or even molecules.
\end{abstract}

DOI: 10.1103/PhysRevX.9.011057

\section{INTRODUCTION}

In quantum simulation and computing, the assembly of large arrays of individually controllable particles is a frontier challenge. Ultracold gases of neutral atoms have long simulated quantum physics on a macroscopic scale, and quantum gas microscopes are now a window to microscopic dynamics $[1,2]$. However, the desire for the control of individual atoms, in particular, for quantum computing, motivates pursuing bottom-up engineering of neutral atom arrays [3-6]. In a Maxwell's demon approach, experimenters image single atoms and subsequently rearrange them into a desired pattern. The resulting ordered arrays present new opportunities in studies of multiparticle quantum dynamics [7-15]. Yet, compared to trapped ions, single neutral atoms are still difficult to trap and assemble.

In our work, we form ordered atom arrays by combining dense loading of large optical-tweezer arrays with atom imaging and rearrangement (Fig. 1). Using $\Lambda$-enhanced

\footnotetext{
*These authors contributed equally.

†regal@colorado.edu
}

Published by the American Physical Society under the terms of the Creative Commons Attribution 4.0 International license. Further distribution of this work must maintain attribution to the author(s) and the published article's title, journal citation, and DOI.
Subject Areas: Atomic and Molecular Physics, Quantum Information gray-molasses $(\Lambda \mathrm{GM})$ on the $\mathrm{D}_{1}$ line of ${ }^{87} \mathrm{Rb}[16,17]$, we can load single atoms with high efficiency in a trap shallower than required for standard sub-Poissonian loading [18] and nearly an order of magnitude shallower than required for previous enhanced loading [19]. While we demonstrate the idea with an array of optical tweezers in 2D, dense loading could also be used in optical lattices or in microtraps in $3 \mathrm{D}[14,15]$. We predict our technique will scale up a neutral-atom array assembly by expanding rearrangement algorithms and by enabling considerably larger ordered arrays.

To isolate single atoms in optical tweezers or lattices, one typically drives light-assisted collisions in the collisional blockade regime using red-detuned light $[18,20]$. In this case, atoms are photoassociated to attractive molecular states in which they accelerate towards each other and gain kinetic energy that predominantly expels both from the trap [Fig. 1(a)]. If the collisions occur quickly enough to dominate the dynamics, as is the case in microtraps, a single atom is left about half of the time. In the pioneering work of Ref. [19], after adding a blue-detuned laser to drive atoms into repulsive molecular states, the energy gained in the collision is tuned to induce single-atom loss [19,21-23]. Loading efficiency is enhanced to $90 \%$, but at the cost of requiring large trap depths $\left(U / k_{B} \sim 3 \mathrm{mK}\right.$ compared to $1 \mathrm{mK}$ for red-detuned loading) and, hence, making the use of the technique untenable in large arrays. 


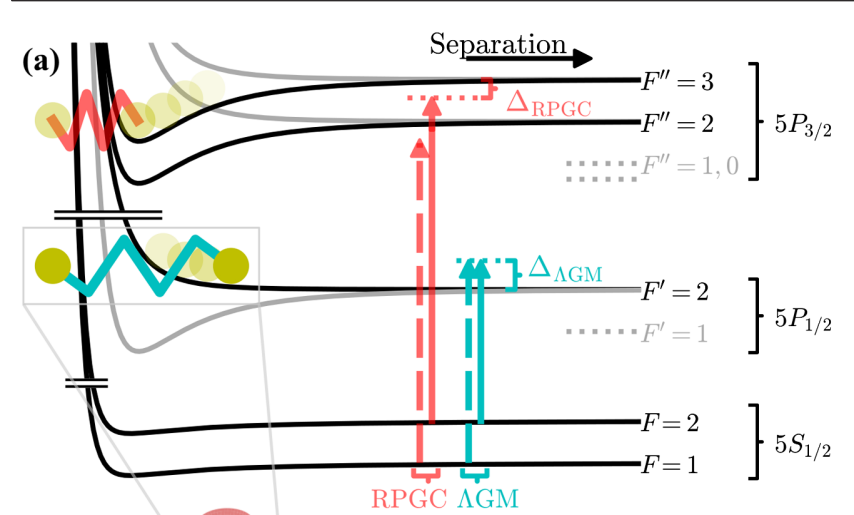

(b)

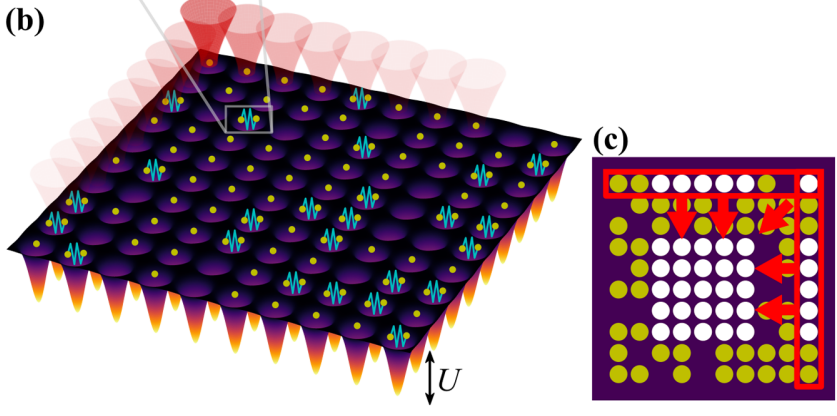

FIG. 1. Enhanced loading and rearrangement in large arrays. (a) Sketch of the laser configuration, and molecular energies versus interatomic separation. Solid (dashed) arrows show cooling (repump) lasers with indicated detunings. (b) Atoms loaded into an array of 100 traps with depth $U$ formed by optical tweezers undergo blue-detuned light-assisted collisions. (c) Schematic of parallel rearrangement to form a defect-free array with target atoms (white) after removing a subset of loaded atoms (yellow).

Here we resolve the conflict that exists in previous work with enhanced loading-namely, that red-detuned cooling drives lossy collisions and competes with desired bluedetuned collisions. By using $\Lambda \mathrm{GM}$, we have the ability to cool into the trap and photoassociate with the same bluedetuned laser [Fig. 1(a)], and we can control the energy that atoms are given in the collision by varying the laser's detuning. Furthermore, we can make use of red-detuned and blue-detuned molecular photoassociation processes at will. In particular, we first modify the atom number distribution in the microtrap with blue-detuned cooling $(\Lambda \mathrm{GM})$. We then apply red-detuned light, which both assures that not more than a single atom remains and, if it remains, images it. The loading behavior studied in a single trap agrees with a model of consecutive light-assisted collisions to repulsive molecular states. Our model further allows us to identify paths to even more efficient singleatom loading.

We find that we can load a single optical tweezer with a trap depth of $U / k_{B}=0.63(6) \mathrm{mK}$ with $89(1) \%$ efficiency and a $10 \times 10$ array with $80.49(6) \%$ efficiency [Fig. 1(b)]. We also demonstrate a proof-of-principle rearrangement technique that relies on the enhanced loading to create a $6 \times 6$ defect-free array using a simplified sequence of parallel moves of entire rows and columns [Fig. 1(c)] [9]. Lastly, we discuss how the efficiency of both this simplified rearrangement as well as atom-by-atom assembly scale exponentially with the initial filling of the array.

\section{LOADING STUDIES AND MODELING}

\section{A. Loading experiments}

Generally, in $\Lambda \mathrm{GM}[16,17]$, the cooling laser is set bluedetuned of a type-II $\left(F^{\prime} \leq F\right)$ transition and in a $\Lambda$ configuration with a coherent repump laser [Fig. 1(a)]. Because of its greater isolation from nearby hyperfine manifolds, we choose to operate the $\Lambda \mathrm{GM}$ detuned from the $5 P_{1 / 2}\left|F^{\prime}=2\right\rangle$ state [in contrast to, e.g., $5 P_{3 / 2}\left|F^{\prime \prime}=2\right\rangle$ ]. Note that we are motivated to use $\Lambda \mathrm{GM}$ mainly as a natural way to bluedetune both cooling and repump lasers, which is a somewhat different motivation than in recent quantum degenerate gas experiments with light atoms and molecules-namely, that gray-molasses works on open transitions and $\Lambda$ enhancement results in lower temperatures [16,17,24-34].

We first present results from loading a single optical tweezer using $\Lambda \mathrm{GM}$ and compare to standard loading using red-detuned polarization gradient cooling (RPGC) [Figs. 2(b) and $2(\mathrm{c})]$. We capture ${ }^{87} \mathrm{Rb}$ atoms in a magneto-optical trap (MOT) and then cool them into a spatially overlapped optical tweezer with depth $U$ with either $\Lambda$ GM or RPGC. After the cooling and loading stage, we apply RPGC with parameters optimized for fluorescence imaging of the atoms. Initially, this imaging quickly removes remaining atom pairs and then images whether a single atom or no atom remains in the trap [Fig. 2(a)]. The procedure is repeated to determine average single-atom loading efficiencies, i.e., the fraction with which a single atom is found after both the loading and imaging stages. See Fig. 2(a) and the Appendix for experimentalsequence timing and details of the imaging analysis. Also, see Fig. 1(a) and the Appendix for detailed laser configurations.

Figures 2(b) and 2(c) show the loading probability $P$ as a function of both laser detuning from the closest atomic freespace resonance and trap depth, for both RPGC and $\Lambda \mathrm{GM}$. With $\Lambda \mathrm{GM}$ we observe $89(1) \%$ loading efficiency at $\left[\Delta_{\Lambda \mathrm{GM}}, U / k_{B}\right]=[45 \mathrm{MHz}, 0.55(5) \mathrm{mK}]$, and we can still load with approximately $80 \%$ efficiency at trap depths of $U / k_{B} \approx 0.27(3) \mathrm{mK}$. These findings are remarkable, as with the same optical power we can load tweezer arrays that are more densely filled and 2-3 times larger compared to RPGC loading. The maximum RPGC loading of 64(1)\% for $\left[\Delta_{\mathrm{RPGC}}, U / k_{B}\right] \approx[-14 \mathrm{MHz}, 1.1(1) \mathrm{mK}]$ is among the highest reported for $\mathrm{RPGC}[9,10,22,23]$. In the simplest picture of RPGC, one expects $50 \%$ loading, but, in agreement with other studies [23], additional processes result in approximately $35 \%$ of the collisions causing only one atom to leave the trap.

A physically rich picture can be gained from studying the detuning dependence of $\Lambda \mathrm{GM}$ loading [Fig. 2(c)]. First, note that the trap light results in an ac-Stark shift 

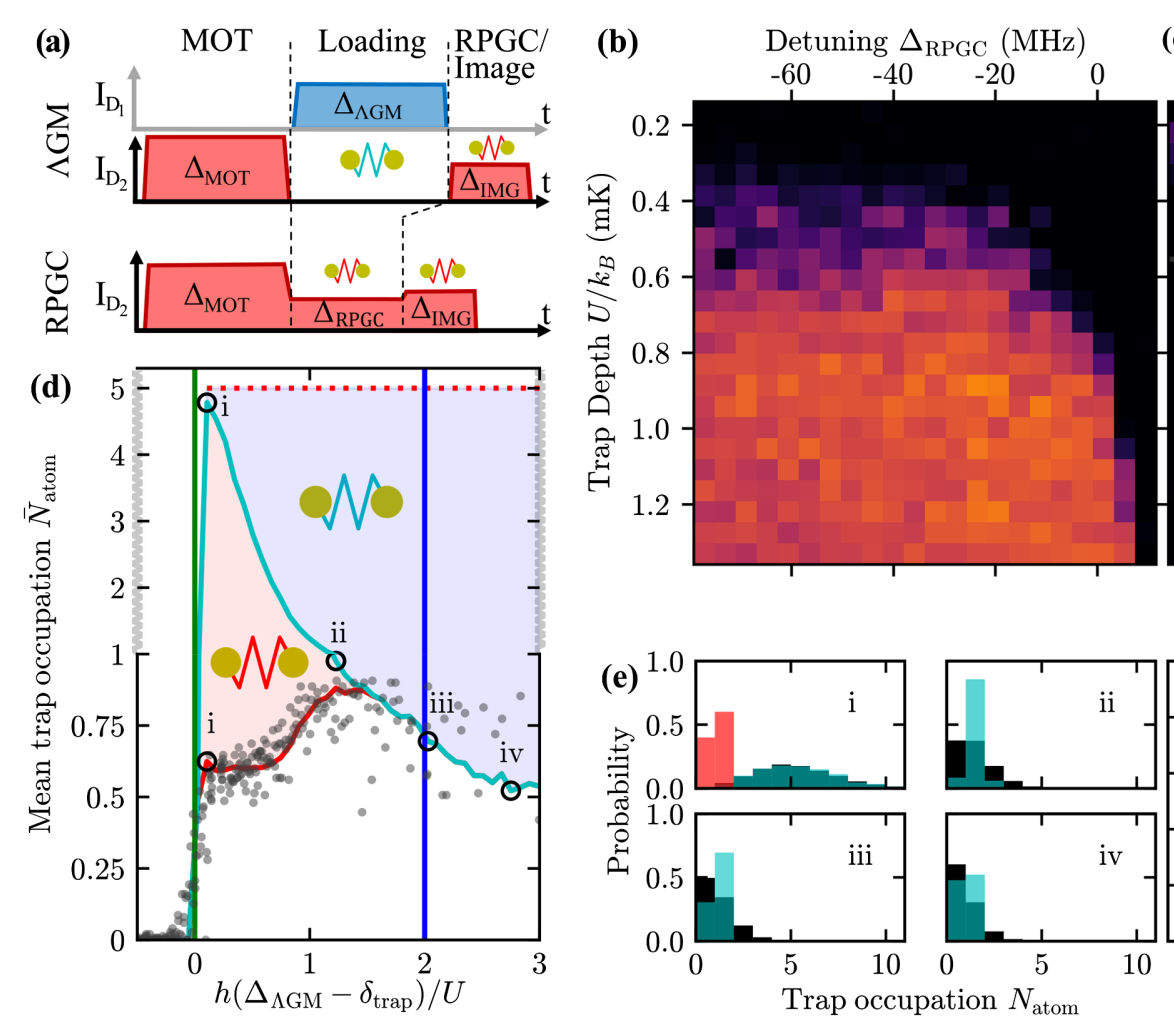

(c) Detuning $\Delta_{\Lambda \mathrm{GM}}(\mathrm{MHz})$
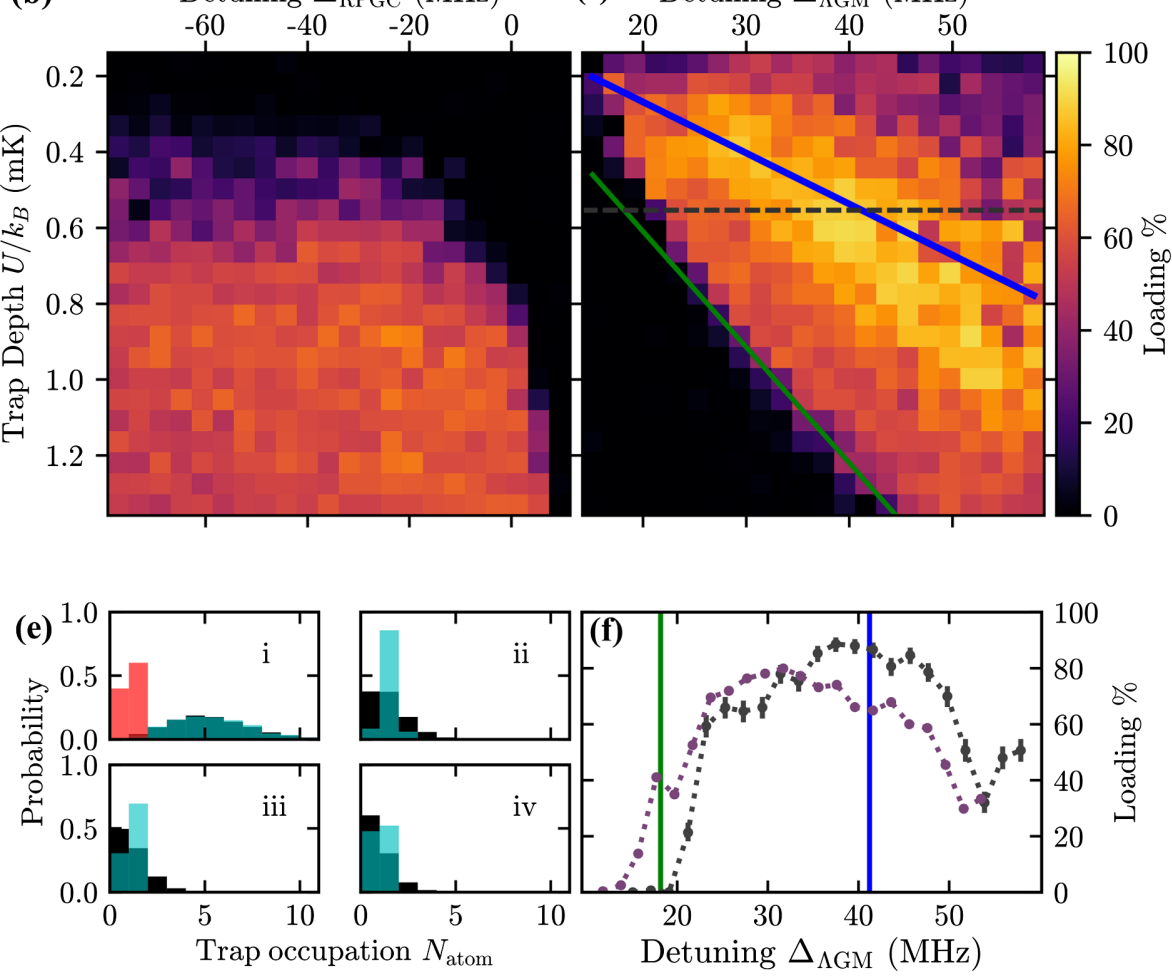

FIG. 2. $\Lambda \mathrm{GM}$ comparative loading studies. (a) Flow diagram of experiments. (b),(c) Average loading efficiency into a single optical tweezer from 150 experiment repetitions as a function of the bare laser detuning from the free-space resonance and trap depth, for RPGC (b) and $\Lambda \mathrm{GM}$ (c). (d) Monte Carlo simulated mean trap occupation with a bilinear vertical scale (black and gray axes). In the $\Lambda \mathrm{GM}$ step, the initial trap occupation (dashed red line) is reduced (cyan line) by blue-detuned collisions (blue area). In the RPGC imaging step, red collisions (red area) further reduce the trap occupation (red line). The resultant is compared with data (gray points) from (c) averaged for $U / k_{B}>0.65 \mathrm{mK}$ (see the text). (e) Histograms (cyan) of the trap occupancy from the Monte Carlo after the $\Lambda$ GM step for detunings indicated by the black circles in (d), compared to a Poisson distribution (black) with the same mean trap occupation, and atom distribution after the RPGC step [red in (i)]. (f) Loading efficiency as a function of $\Lambda$ GM-laser detuning $\Delta_{\text {LGM }}$ for a single tweezer (black) [see also the cut along the black dashed line in (c)] and a regular array of 100 tweezers (purple) at $U / k_{B} \approx 0.55(5) \mathrm{mK}$. Error bars indicate the statistical $1 \sigma$-confidence interval (see the Appendix). Throughout the panels, green lines are the ac-Stark-shifted $|F=2\rangle-\left|F^{\prime \prime}=3\right\rangle$ transition (for RPGC) and ac-Stark-shifted $|F=2\rangle-\left|F^{\prime}=2\right\rangle$ transition $\delta_{\text {trap }}$ (for $\Lambda$ GM), and blue lines are $\delta_{\text {trap }}+2 U / h$.

$\delta_{\text {trap }}=32.8(\mathrm{MHz} / \mathrm{mK})\left(U / k_{B}\right)$ of the atomic transition in the center of the trap [green lines in Figs. 2(c), 2(d), and 2(f)]. The blue line in Figs. 2(c), 2(d), and 2(f), which marks a shift of $2 U / h$ from the trap-shifted resonance, is a key energy scale for the physics of the enhanced loading. At shifts smaller than $2 U / h$, the collision does not give a pair of zero-temperature atoms sitting at the bottom of the trap enough energy for either to escape, while at larger detunings both atoms are expelled. A finite temperature, and hence an initial center of mass motion, blurs the transition and indeed is necessary for inducing the desired single-atom loss. Although our data are roughly consistent with this picture, we look more closely by plotting the data in Fig. 2(c) against a dimensionless detuning $h\left(\Delta_{\Lambda \mathrm{GM}}-\delta_{\text {trap }}\right) / U$. We do this for all data traces $U / k_{B} \geq 0.65 \mathrm{mK}$ [Fig. 2(d)] and observe a number of interesting features. For example, we observe an approximately $60 \%$ loading probability for small detunings and that the maximum loading peaks below the $2 U / h$ shift (blue line).

\section{B. Model}

To elucidate detailed trends, we carry out a Monte Carlo calculation of the collision dynamics. Most generally, we expect loading to be affected by both collisions and the $\Lambda \mathrm{GM}$ cooling performance, and both may be influenced by the nontrivial light shifts and polarization gradients in the tweezer traps. Modeling the interplay of these effects is beyond the scope of this work, but we can understand the collisional process quantitatively if we assume that the continuous $\Lambda \mathrm{GM}$ cooling can load at least a few atoms per trap and rethermalizes any atoms remaining after a collision. The simulation starts by preparing a Poisson-distributed number of atoms $N_{\text {atom }}$ with a mean number $\bar{N}_{\text {atom }}=5$ and temperature $T$, where $\bar{N}_{\text {atom }}$ is chosen $>2.5$ to avoid loading zero atoms initially. To simulate the finite experiment cooling time, we calculate a finite number of 5000 time steps, each having two atoms collide once if they are closer than $100 \mathrm{~nm}$. A collision might eject none, one, or both 
atoms out of the trap, depending on the final energy of each atom, which is determined by their precollision energy and the collisional energy gain $E=h\left[\Delta_{\Lambda \mathrm{GM}}-\delta_{\text {trap }}\right]$. This process continually reduces $N_{\text {atom }}$ in each time step. At the end, the RPGC imaging is simulated by assuming that it entails a fast collisional process at the start of the image, where red-detuned collisions reduce atom numbers in a manner consistent with our red loading-namely, we reduce any remaining $N_{\text {atom }}>1$ by 2 with a chance of $65 \%$ and by 1 with a chance of $35 \%$ until $N_{\text {atom }} \leq 1$.

Figure 2(d) shows the result of the Monte Carlo simulation by indicating the mean trap occupation $\bar{N}_{\text {atom }}$ as a function of the normalized collisional energy gain. During $\Lambda \mathrm{GM}$ loading, the initial atom number (red dashed line) is reduced (cyan line). During RPGC imaging, $\bar{N}_{\text {atom }}$ is further reduced [red line in Fig. 2(d)]. Figure 2(e) shows the simulated atom-number distribution $\left(N_{\text {atom }}\right)$ in the trap and how atom loss in the $\Lambda \mathrm{GM}$ and RPGC phase modifies the Poisson distribution.

We observe three physical regimes: For $E \ll 2 U$ [see (i) in Fig. 2(e)], the $\Lambda$ GM phase has little effect as almost no atom loss occurs, and, hence, the initial Poisson distribution (black distribution) is not modified (blue distribution). The initial phase of the RPGC-imaging step, however, reduces the number of atoms to 0 or 1 , yielding a RPGC-like $65 \%$ mean trap occupation (red distribution). In contrast, for $E \gg 2 U$ (iv), two-body losses dominate in the $\Lambda \mathrm{GM}$ phase, as every collision expels both atoms from the trap, resulting in $N_{\text {atom }}=0\left(N_{\text {atom }}=1\right)$ in approximately $50 \%$ of the cases if the loaded $N_{\text {atom }}$ is even (odd). Hence, after $\Lambda$ GM, $N_{\text {atom }}<2$ and the red-detuned imaging phase does not modify the atom-number distribution anymore. At the transition $E \approx 2 U$ (iii), both single-atom and two-body losses occur in the $\Lambda \mathrm{GM}$ phase with roughly equal probability because of the finite temperature. Since twobody losses tend toward an equal distribution of $N_{\text {atom }}=0$ and 1 and single-atom loss toward $N_{\text {atom }}=1$, we load a single atom $\left(N_{\text {atom }}=1\right)$ in $75 \%$ of the cases. Again, RPGC imaging does not modify the distribution, as $N_{\text {atom }}<2$ after $\Lambda \mathrm{GM}$. Maximal loading probability is found at $E<2 U$ (ii) where only single-atom loss occurs. Here, any occurrence of $N_{\text {atom }}=0$ is a result of either no atoms having been loaded initially or the $\Lambda \mathrm{GM}$ step not being finished (finite $N_{\text {atom }}>1$ after $\Lambda \mathrm{GM}$ ), and RPGC imaging then ejects pairs of atoms.

Our model indicates no fundamental limitations to the loading efficiency and that, by optimizing the trap size, atom temperature, and related parameters, it may be possible to reach higher loading fractions. Note that the only free parameter that affects the prediction of the simulation is the atom temperature $T$ in the trap. The simulation describes our data well for $T=120(10) \mu \mathrm{K}$, which needs to be understood as an average value for the different trap depths $U$ that are investigated. This value is close to the free-space $\Lambda \mathrm{GM}$ temperature we measure of $T \approx 50 \mu \mathrm{K}$, which is higher than typical values, likely due to nonideal beam geometries (see the Appendix).

\section{IMPACT OF GRAY-MOLASSES LOADING ON ARRAY ASSEMBLY}

\section{A. Loading in large arrays}

We also perform a loading study for an array of $10 \times 10$ optical tweezers spaced by $2 \mu \mathrm{m}$. We display the measurement at $U / k_{B}=0.55(5) \mathrm{mK}$ as the purple line in Fig. 2(f). Compared to the single-trap data at similar $U$ (black), the data are shifted to smaller detunings, and we observe a maximum loading of $80.49(6) \%$ in a single run averaged over the $10 \times 10$ array. These effects could be due to a variety of consequences of the larger array: variations in the trap shape and depth or overall degradation of the optical spot sizes (see the Appendix). Note that our experimental apparatus is designed and optimized to entangle closely spaced atoms in ground states, in contrast to systems that interface (farther-spaced) Rydberg atoms. This design places constraints on the tweezer array, such as acousto-optic device mode, trap-light detuning, optical power, and high-NA field of view, that mean with typical loading we are limited to working with array sizes less than $10 \times 10$. However, $\Lambda \mathrm{GM}$ loading allows us to scale up both the size of our arrays as well as the total number of atoms we can trap and is a unique realization for enhanced loading in optical tweezers.

\section{B. Rearrangement example}

One prospect for dense loading is that it opens up new possibilities for rearrangement algorithms for array assembly. Here, we present one particular experimental example. After densely loading an array, we first obtain the location of each atom using a single image [left panel in Fig. 3(a)]. Even with dense loading, the probability of loading a specific set of $6 \times 6$ traps is exponentially small [dashed lines in Fig. 3(b)]. However, there are many potential sets of (sometimes disjoint) $6 \times 6$ traps embedded in the $10 \times 10$ array. We then search for such a configuration of completely loaded $6 \times 6$ traps. If successful, we turn off the extra traps to remove the excess atoms and then contract and shift the identified disjoint array in a single move [(right panel) in Fig. 3(b)] [9]. Currently, successful rearrangement to a square $n=36$ ( 6 by 6 ) array works in only $0.1 \%$ of cases due to unexpected loss observed when turning off rows and columns, in which an atom is effectively lost with a 17\% chance. This loss is technical in nature, and potential causes include intermodulation in the tweezer-generating orf tones or collisions between the trapped and dropped atoms. But, as illustrated in Fig. 3(b), observing this array with this parallel technique would have been impossible without enhanced loading. In increasing the loading probability $P$ from $60 \%$ to $80 \%$, the percentage of experiment runs in which one could possibly extract a 

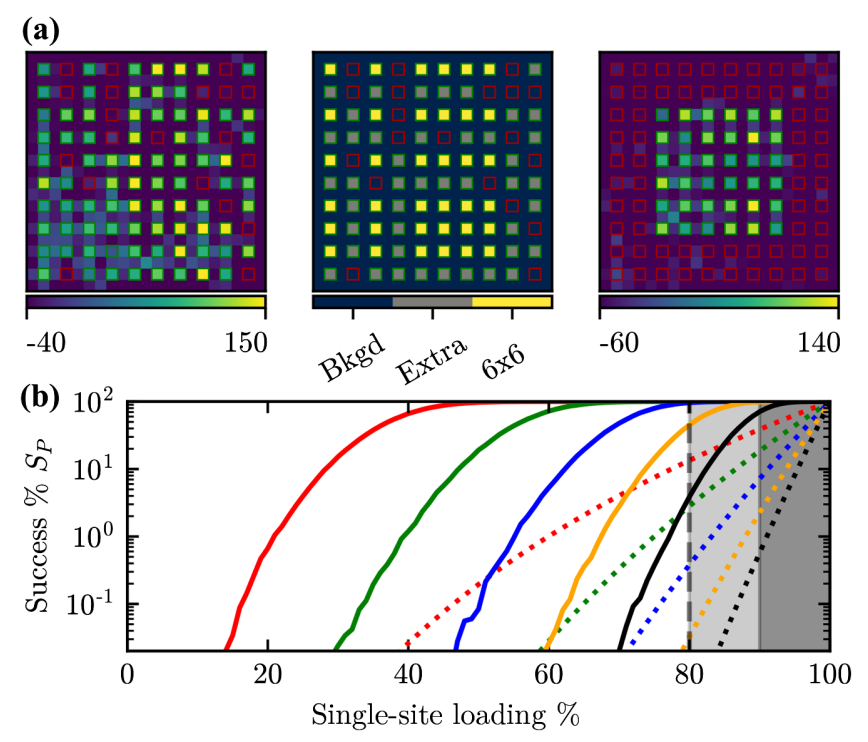

FIG. 3. Parallel rearrangement in 2D. (a) Single image of 80 atoms in an array of 100 traps with a separation of $2 \mu \mathrm{m}$ (left). Binarized loading (center) indicating empty traps (red squares), occupied traps (gray pixels), and loaded traps selected for parallel rearranging (yellow pixels) to a defect-free $6 \times 6$ array. For the single-shot images, in each trap pixel (background pixel) the atom count threshold (average threshold of nearest neighbors) is subtracted for clarity from the count number recorded by the camera (see the Appendix). (b) Monte Carlo simulated success probabilities $\left(S_{P}\right)$ of finding a filled disjoint array of atoms within $10 \times 10$ traps as a function of loading efficiencies (solid lines), and the corresponding loading probability of a specific array without rearrangement (dashed lines). Colors indicate target array sizes: $3 \times 3$ (red), $4 \times 4$ (green), $5 \times 5$ (blue), $6 \times 6$ (orange), and $7 \times 7$ (black). The light gray area is accessible only with an average loading greater than $80 \%$, and the dark gray area for greater than $90 \%$.

defect-free $6 \times 6$ array goes from $0.02 \%$ to $37 \%$. Notably, this entire procedure is completed using only a pair of acousto-optic modulators to control the optical tweezers.

\section{Scaling arguments}

The full potential of dense loading using $\Lambda \mathrm{GM}$ comes when combined with the most advanced atom-by-atom rearrangement algorithms in 2D or 3D [10,14,15]. In principle, if the Maxwell's demon atom rearrangement operation is perfect, and if a large number of traps are used, one would expect that very large arrays could be created through a bottom-up approach regardless of the loading. Practically, however, there are many factors that steeply limit creating large-scale systems with optical tweezer assembly - in particular, a finite atom lifetime compared to the time required for rearrangement and also simply the number of traps that can be created and loaded.

Our approach addresses these problems because it is efficient in shallow traps. Hence, with $\Lambda \mathrm{GM}$, more loadable traps can be created with the same amount of optical power.
Additionally, 2D algorithms fill defects in a target array of size $n$ with a sequence of $m \propto(1-P) n^{1.4}$ single moves, which we verify with Monte Carlo simulations [10]. In scaling up array sizes, the time and number of moves required become lengthy, lowering the probability of successful rearrangement $\left(S_{P}\right)$, as errors $\epsilon$ due to finite move fidelities and background collisions suppress this success rate as $e^{-m \epsilon}[10]$. Increasing the loading probability $P$ from $P=60 \%$ to $P=90 \%$ decreases $m$ by a factor of 4 , making larger array sizes more obtainable and exponentially improving the success probability $S_{P}$.

\section{CONCLUSION}

In conclusion, by gaining control over photoassociation to molecular states, we have demonstrated enhanced loading of arrays of shallow optical tweezers. As described in Secs. III A and III B, we achieved a strong relative improvement on our trapped atom numbers, but experimental platforms designed to host more optical traps than our system will stand to benefit even more. For example, Ref. [10] loads approximately 50 atoms with a 2D array of 100 traps of $1 \mathrm{mK}$ depth. With the same optical power and $\Lambda \mathrm{GM}$, one would expect to utilize 370 traps of $0.27 \mathrm{mK}$ depth and, based on the shallow-depth loading of single atoms at $P=80 \%$ in Fig. 2(c), load approximately 300 atoms - a sixfold increase. Furthermore, the density of the filling will affect the number of moves required in rearrangement [9]. Using a technique that moves atoms individually [10], our Monte Carlo simulations indicate that rearranging 300 atoms at $P=50 \%$ requires approximately 900 moves on average, whereas at $P=80 \%$ it requires 320 moves. As a result, the probability to retain all 300 atoms in the rearrangement protocol increases roughly from $0.1 \%$ to $10 \%$ when going from $P=50 \%$ to $P=80 \%$, assuming a $420 \mathrm{~s}$ atom lifetime [35], $1 \mathrm{~ms}$ per move, and a $99.3 \%$ move fidelity [10].

While we have studied one particular blue-detuned cooling mechanism- $\Lambda \mathrm{GM}$ on the ${ }^{87} \mathrm{Rb} 5 S_{1 / 2}-5 P_{1 / 2}$ transition-it will be interesting to explore a variety of other related cooling techniques in future experiments. In particular, it is also known that gray-molasses is effective on the $5 S_{1 / 2}-5 P_{3 / 2}$ transition [33], and future studies could compare the salient molecular physics in each manifold [36]. Furthermore, we expect that our work will be the start of explorations of the interplay of collisions and cooling in microtraps for a host of blue-detuned cooling mechanisms with alkali atoms, other atomic species, and even molecules [30,37].

\section{ACKNOWLEDGMENTS}

We thank Yiheng Lin, Kai-Niklas Schymik, Ludovic Brossard, Junling Long, and Brian Lester for technical assistance and Jose D'Incao, Paul Julienne, Ana Maria Rey, and Adam Kaufman for helpful comments and fruitful 
discussion. We acknowledge funding from ONR Grant No. N00014-17-1-2245, NSF Grant No. PHYS 1734006, the Cottrell Scholars program, and the David and Lucile Packard Foundation. M. O. B. acknowledges support from an NDSEG Fellowship. T. T. acknowledges funding from SNF under Project No. P2EZP2_172208.

\section{APPENDIX A: OPTICAL TWEEZERS}

We generate an array of optical-tweezer traps spaced by $2 \mu \mathrm{m}$ in the $x y$ plane by passing a single $850 \mathrm{~nm}$ laser beam through two orthogonal longitudinal-wave $\mathrm{TeO}_{2}$ acoustooptical modulators (AOMs) with center frequencies (bandwidths) of $180 \mathrm{MHz}(90 \mathrm{MHz})$. Each modulator is driven with a sum of radio-frequency (rf) tones with a frequency (amplitude) that can be individually and dynamically adjusted to control the position (intensity) of different tweezer rows and columns. The relative phases of the tones are set to minimize intermodulation in the rf setup. The array of deflections created by the AOMs is then imaged by a 0.6 NA objective lens into a glass cell. This imaging creates a trap with a $0.68 \mu \mathrm{m}$ waist for a single tweezer and traps with an average waist of $0.75 \mu \mathrm{m}$ for a $10 \times 10$ array. The standard deviation of the trap depths is minimized to $8 \%$ by optimizing the rf amplitudes. Trap depths are calibrated by measuring light shifts of in-trap atomic transitions as a function of the trap power and applying a linear fit; the slope gives a calibration of the intensity of trap light the atom experiences, which can be used to directly calculate the trap depth [38]. Errors on trap depths are $1 \sigma$ errors extrapolated from the errors on the slope of the linear fit. The lifetime of atoms in the traps is limited to $5 \mathrm{~s}$ by the background pressure.

\section{APPENDIX B: LASER COOLING AND LOADING}

In all experiments, three beam paths are used to address the atoms. Two (diagonal) paths are along the diagonals of the $x y$ plane, and a third (acute) path in the $x z$ plane is at an angle of $55^{\circ}$ from the $z$ axis to avoid the objective [39]. All lasers along these paths are retroreflected and in a $\sigma^{+} \sigma^{-}$ polarization configuration.

Our MOT is spatially overlapped with the trap array and cools atoms for $500 \mathrm{~ms}$ to a temperature of approximately $100 \mu \mathrm{K}$, measured by imaging its ballistic expansion. The cooling (repump) laser is red-detuned from the $D_{2}$ $|F=2\rangle \rightarrow\left|F^{\prime \prime}=3\right\rangle\left(|F=1\rangle \rightarrow\left|F^{\prime \prime}=2\right\rangle\right)$ transition and applied on all three beam paths (on only the diagonal paths). In the case of the 20-ms-long RPGC stage, we cool the atoms to approximately $10 \mu \mathrm{K}$. For this process, we detune the cooling (repump) laser by $\Delta_{\mathrm{RPGC}}(20 \mathrm{MHz})$, set the intensities at $1.3 I_{\text {sat }}\left(0.1 I_{\text {sat }}\right)$ on the diagonal paths and $4.5 I_{\text {sat }}$ $\left(0 I_{\text {sat }}\right)$ on the acute path, and zero the magnetic fields.

In the case of the 200-ms-long $\Lambda$ GM stage, we apply a cooling laser that is detuned by $\Delta_{\Lambda \mathrm{GM}}$ from the $\mathrm{D}_{1}$
$|F=2\rangle \rightarrow\left|F^{\prime}=2\right\rangle$ transition at $2.5 I_{\text {sat }}\left(0.4 I_{\text {sat }}\right)$ on the acute (diagonal) paths. We create the coherent repump beam from the cooling laser on the acute path using an electro-optic modulator. The repump beam is detuned by $\Delta_{\Lambda \mathrm{GM}}+0.14 \mathrm{MHz}$ from the $\mathrm{D}_{1}|F=1\rangle \rightarrow\left|F^{\prime}=2\right\rangle$ transition and at $1.5 I_{\text {sat }}$. Note that the optimal $\Lambda \mathrm{GM}$ free-space temperature of $50 \mu \mathrm{K}$ is reached for $\Delta_{\Lambda \mathrm{GM}} \approx 15 \mathrm{MHz}$ and is likely limited by the beam path geometry and repump light configuration.

\section{APPENDIX C: IMAGING, DATA, AND STATISTICS}

Regardless of the loading configuration, we image the atoms using another RPGC stage with the cooling beam $\Delta_{\mathrm{RPGC}}=-19 \mathrm{MHz}$ at $3 I_{\text {sat }}$ only on the acute path. We alternate the tweezer light with the imaging light at $2 \mathrm{MHz}$ to scatter light when atoms are experiencing no light shifts. This configuration is maintained for $20 \mathrm{~ms}$, during which we collect scattered photons on an EMCCD camera, superbinned to $4 \times 4$ pixels to reduce readout noise. As we now discuss experimental evidence for, this red-detuned imaging process quickly kicks out any pairs of atoms that might exist, for example, in the case of a gray-molasses loading stage with a small detuning [Figs. 2(d) and 2(e)]. Accordingly, it does not resolve a tweezer's occupation number following the gray-molasses stage [illustrated by the cyan line in Fig. 2(d)] but rather maps the atom number onto 0 or 1 . If this loss does not occur quickly compared to the imaging time, we sometimes collect numbers of photons significantly larger than our calibrated single-atom scattering rate. We do not observe this signature despite high experimental statistics, suggesting a subpercent impact of these effects on the imaging.

At every atom location individually, to determine a count threshold that indicates the presence of an atom in the trap, we create a histogram of all counts during an experiment and fit it with a sum of two Gaussians. The threshold with maximal fidelity $\mathcal{F}$ is found, where $\mathcal{F}=1-\left(E_{f p}+E_{f n}\right)$, with $E_{f p}\left(E_{f n}\right)$ being the expected rate of false positives (false negatives) from the fits. This process converts a sequence of counts to a sequence of Booleans which is averaged to determine the loading probability. By finding thresholds for each trap individually and subtracting them from the images in Fig. 3(a), we compensate for a spatially varying background noise and, due to the limited field of view of our high-NA lens, the different numbers of photons we collect for each trap.

All errors reported indicate $1 \sigma$ equal-tailed Jeffrey's prior confidence intervals [40]. The loading efficiencies reported in the main text for RPGC [64(1)\%], $\Lambda$ GM [89(1)\%], and $10 \times 10-\Lambda \mathrm{GM}[80.49(6) \%]$ are obtained by analyzing 2000-, 1000-, and 5000-per-atom repetitions with threshold fidelities $0.987,0.998$, and 0.993 , respectively. 
[1] W. S. Bakr, J. I. Gillen, A. Peng, S. Fölling, and M. Greiner, A Quantum Gas Microscope for Detecting Single Atoms in a Hubbard-Regime Optical Lattice, Nature (London) 462, 74 (2009).

[2] J. F. Sherson, C. Weitenberg, M. Endres, M. Cheneau, I. Bloch, and S. Kuhr, Single-Atom-Resolved Fluorescence Imaging of an Atomic Mott Insulator, Nature (London) 467, 68 (2010).

[3] L. Isenhower, E. Urban, X. L. Zhang, A. T. Gill, T. Henage, T. A. Johnson, T. G. Walker, and M. Saffman, Demonstration of a Neutral Atom Controlled-Not Quantum Gate, Phys. Rev. Lett. 104, 010503 (2010).

[4] T. Wilk, A. Gaëtan, C. Evellin, J. Wolters, Y. Miroshnychenko, P. Grangier, and A. Browaeys, Entanglement of Two Individual Neutral Atoms Using Rydberg Blockade, Phys. Rev. Lett. 104, 010502 (2010).

[5] A. M. Kaufman, B. J. Lester, C. M. Reynolds, M. L. Wall, M. Foss-Feig, K. R. A. Hazzard, A. M. Rey, and C. A. Regal, Two-Particle Quantum Interference in TunnelCoupled Optical Tweezers, Science 345, 306 (2014).

[6] A. M. Kaufman, B. J. Lester, M. Foss-Feig, M. L. Wall, A. M. Rey, and C. A. Regal, Entangling Two Transportable Neutral Atoms via Local Spin Exchange, Nature (London) 527, 208 (2015).

[7] D. S. Weiss, J. Vala, A. V. Thapliyal, S. Myrgren, U. Vazirani, and K. B. Whaley, Another Way to Approach Zero Entropy for a Finite System of Atoms, Phys. Rev. A 70, 040302(R) (2004).

[8] Y. Miroshnychenko, W. Alt, I. Dotsenko, L. Förster, M. Khudaverdyan, D. Meschede, D. Schrader, and A. Rauschenbeutel, Quantum Engineering: An Atom-Sorting Machine, Nature (London) 442, 151 (2006).

[9] M. Endres, H. Bernien, A. Keesling, H. Levine, E. R. Anschuetz, A. Krajenbrink, C. Senko, V. Vuletic, M. Greiner, and M. D. Lukin, Atom-by-Atom Assembly of Defect-Free One-Dimensional Cold Atom Arrays, Science 354, 1024 (2016).

[10] D. Barredo, S. de Léséleuc, V. Lienhard, T. Lahaye, and A. Browaeys, An Atom-by-Atom Assembler of Defect-Free Arbitrary Two-Dimensional Atomic Arrays, Science 354, 1021 (2016).

[11] H. Bernien, S. Schwartz, A. Keesling, H. Levine, A. Omran, H. Pichler, S. Choi, A. S. Zibrov, M. Endres, M. Greiner, V. Vuletić, and M. D. Lukin, Probing Many-Body Dynamics on a 51-Atom Quantum Simulator, Nature (London) 551, 579 (2017).

[12] M. Marcuzzi, J. Minář, D. Barredo, S. de Léséleuc, H. Labuhn, T. Lahaye, A. Browaeys, E. Levi, and I. Lesanovsky, Facilitation Dynamics and Localization Phenomena in Rydberg Lattice Gases with Position Disorder, Phys. Rev. Lett. 118, 063606 (2017).

[13] W. Lee, H. Kim, and J. Ahn, Defect-Free Atomic Array Formation Using the Hungarian Matching Algorithm, Phys. Rev. A 95, 053424 (2017).

[14] D. Barredo, V. Lienhard, S. de Léséleuc, T. Lahaye, and A. Browaeys, Synthetic Three-Dimensional Atomic Structures Assembled Atom by Atom, Nature (London) 561, 79 (2018).

[15] A. Kumar, T.-Y. Wu, F. Giraldo, and D. S. Weiss, Sorting Ultracold Atoms in a Three-Dimensional Optical Lattice in a Realization of Maxwell's Demon, Nature (London) 561, 83 (2018).
[16] A. T. Grier, I. Ferrier-Barbut, B. S. Rem, M. Delehaye, L. Khaykovich, F. Chevy, and C. Salomon, $\Lambda$-Enhanced Sub-Doppler Cooling of Lithium Atoms in D $D_{1}$ Gray Molasses, Phys. Rev. A 87, 063411 (2013).

[17] D. R. Fernandes, F. Sievers, N. Kretzschmar, S. Wu, C. Salomon, and F. Chevy, Sub-Doppler Laser Cooling of Fermionic $40 \mathrm{~K}$ Atoms in Three-Dimensional Gray Optical Molasses, Europhys. Lett. 100, 63001 (2012).

[18] N. Schlosser, G. Reymond, I. Protsenko, and P. Grangier, Sub-Poissonian Loading of Single Atoms in a Microscopic Dipole Trap, Nature (London) 411, 1024 (2001).

[19] T. Grünzweig, A. Hilliard, M. McGovern, and M. F. Andersen, Near-Deterministic Preparation of a Single Atom in an Optical Microtrap, Nat. Phys. 6, 951 (2010).

[20] N. Schlosser, G. Reymond, and P. Grangier, Collisional Blockade in Microscopic Optical Dipole Traps, Phys. Rev. Lett. 89, 023005 (2002).

[21] A. V. Carpentier, Y. H. Fung, P. Sompet, A. J. Hilliard, T. G. Walker, and M. F. Andersen, Preparation of a Single Atom in an Optical Microtrap, Laser Phys. Lett. 10, 125501 (2013).

[22] B. J. Lester, N. Luick, A. M. Kaufman, C. M. Reynolds, and C. A. Regal, Rapid Production of Uniformly Filled Arrays of Neutral Atoms, Phys. Rev. Lett. 115, 073003 (2015).

[23] Y. H. Fung and M. F. Andersen, Efficient Collisional Blockade Loading of a Single Atom into a Tight Microtrap, New J. Phys. 17, 073011 (2015).

[24] A. Aspect, E. Arimondo, R. Kaiser, N. Vansteenkiste, and C. Cohen-Tannoudji, Laser Cooling Below the One-Photon Recoil Energy by Velocity-Selective Coherent Population Trapping, Phys. Rev. Lett. 61, 826 (1988).

[25] M. S. Shahriar, P. R. Hemmer, M. G. Prentiss, P. Marte, J. Mervis, D. P. Katz, N. P. Bigelow, and T. Cai, Continuous Polarization-Gradient Precooling-Assisted Velocity-Selective Coherent Population Trapping, Phys. Rev. A 48, R4035 (1993).

[26] G. Grynberg and J.-Y. Courtois, Proposal for a MagnetoOptical Lattice for Trapping Atoms in Nearly-Dark States, Europhys. Lett. 27, 41 (1994).

[27] T. Esslinger, F. Sander, A. Hemmerich, T. W. Hänsch, H. Ritsch, and M. Weidemüller, Purely Optical Dark Lattice, Opt. Lett. 21, 991 (1996).

[28] D. J. McCarron, E. B. Norrgard, M. H. Steinecker, and D. DeMille, Improved Magneto-Optical Trapping of a Diatomic Molecule, New J. Phys. 17, 035014 (2015).

[29] J. A. Devlin and M. R. Tarbutt, Three-Dimensional Doppler, Polarization-Gradient, and Magneto-Optical Forces for Atoms and Molecules with Dark States, New J. Phys. 18, 123017 (2016).

[30] L. W. Cheuk, L. Anderegg, B. L. Augenbraun, Y. Bao, S. Burchesky, W. Ketterle, and J. M. Doyle, $\Lambda$-Enhanced Imaging of Molecules in an Optical Trap, Phys. Rev. Lett. 121, 083201 (2018).

[31] J. Lim, J. R. Almond, M. A. Trigatzis, J. A. Devlin, N. J. Fitch, B. E. Sauer, M. R. Tarbutt, and E. A. Hinds, Laser Cooled YbF Molecules for Measuring the Electron's Electric Dipole Moment, Phys. Rev. Lett. 120, 123201 (2018).

[32] L. Anderegg, B. L. Augenbraun, Y. Bao, S. Burchesky, L. W. Cheuk, W. Ketterle, and J. M. Doyle, Laser Cooling of Optically Trapped Molecules, Nat. Phys. 14, 890 (2018). 
[33] S. Rosi, A. Burchianti, S. Conclave, D. S. Naik, G. Roati, C. Fort, and F. Minardi, $\Lambda$-Enhanced Grey Molasses on the $\mathrm{D}_{2}$ Transition of Rubidium-87 Atoms, Sci. Rep. 8, 1301 (2018).

[34] G. D. Bruce, E. Haller, B. Peaudecerf, D. A. Cotta, M. Andia, S. Wu, M. Y. H. Johnson, B. W. Lovett, and S. Kuhr, Sub-Doppler Laser Cooling of $40 \mathrm{~K}$ with Raman Gray Molasses on the $D_{2}$ Line, J. Phys. B 50, 095002 (2017).

[35] J. P. Covey, I. S. Madjarov, A. Cooper, and M. Endres, 2000-Times Repeated Imaging of Strontium Atoms in ClockMagic Tweezer Arrays, arXiv:1811.06014.

[36] J. Weiner, V.S. Bagnato, S. Zilio, and P. S. Julienne, Experiments and Theory in Cold and Ultracold Collisions, Rev. Mod. Phys. 71, 1 (1999).
[37] L. R. Liu, J. D. Hood, Y. Yu, J. T. Zhang, N. R. Hutzler, T. Rosenband, and K.-K. Ni, Building One Molecule from a Reservoir of Two Atoms, Science 360, 900 (2018).

[38] F. L. Kien, P. Schneeweiss, and A. Rauschenbeutel, Dynamical Polarizability of Atoms in Arbitrary Light Fields: General Theory and Application to Cesium, Eur. Phys. J. D 67, 92 (2013).

[39] A. M. Kaufman, Laser-Cooling Atoms to Indistinguishability: Atomic Hong-Ou-Mandel Interference and Entanglement through Spin Exchange, Ph.D. thesis, University of Colorado at Boulder, 2015.

[40] L. D. Brown, T. T. Cai, and A. DasGupta, Interval Estimation for a Binomial Proportion, Stat. Sci. 16, 101 (2001). 